\title{
ANALYSIS OF AN ARBITRARY PROFILE REFLECTOR ANTENNA HAVING RESISTIVE-TYPE SURFACE - H-POLARIZATION CASE
}

\author{
Taner Ŏguzer ${ }^{1}$, Ayhan Altintas ${ }^{2}$ and A.I. Nosich ${ }^{3}$ \\ ${ }^{1}$ Department of Electrical and Electronics Eng., Dokuz Eylül University, 35160 Buca, İzmir, Turkey \\ ${ }^{2}$ Department of Electrical and Electronics Eng., Bilkent University, 06800 Ankara, Turkey \\ ${ }^{3}$ Institute of Radio-Physics and Electronics, National Academy of Sciences, Kharkov 61085, Ukraine
}

\begin{abstract}
A regularization-based numerical solution is obtained for arbitrary-shape conic section profile reflector antenna in 2-D, for the H-polarization case. New point is that the reflector surface is assumed a resistive-type material. The problem is treated by reducing the singular integral equation obtained from the boundary condition to the dual series equations and application of the Riemann Hilbert Problem (RHP) technique. The resulting matrix equation has regularized form. Sample numerical results are obtained for various values of the eccentricity of the conic section contour of reflector and the resistivity of its surface.
\end{abstract}

1. INTRODUCTION: Reflector antennas have originally 3-D geometry but sometimes nearly 2-D cylindrical reflectors are also used in practice; besides, these 2-D reflectors can be considered as canonical shapes to validate the numerical analysis methods. In real applications large dishes are mostly used and can be simulated by high-frequency ray-tracing techniques like GTD, UTD, PO and PTD. However, these techniques do not produce a single solution valid in all directions. Still they are erroneous in the caustic and transition regions. In the simulations of 2-D reflectors, Wiener-Hopf method based analytical treatment has been applied and certain asymptotic results have been obtained in [1], however they have a limited validity. On the other hand purely numerical techniques can also be applied to the reflector antenna problems. Method-of-Moments (MoM) with local basis functions is a standard tool in electromagnetics, however it is efficient only for small or medium size single-reflector geometries. Moreover, MoM does not guarantee the accuracy and the convergence of the performed numerical solution.

An alternative for the same 2-D reflector antenna problem is the method of analytical regularization (MAR) [2]. With MAR, a part of the full-wave operator is inverted analytically and the resulting operator equation having favorable features is solved numerically. RHP based solutions are examples of the MAR. In [3], RHP based solutions were built for the analysis of the circular-strip 2-D reflector antenna problems for the both polarizations. These RHP based solutions provide fast convergence and easily controlled accuracy. In [4] and [5], the conic-section-profile reflectors excited by complex-source-point (CSP) feeds were analyzed and the regularized solutions were obtained. Similar MAR-type algorithms have been also proposed based on different techniques. For example, projection on Chebyshev polynomials and Jacobi polynomials was suggested in [6] and [7], respectively. However these papers did not present reliable numerical data for arbitrary profiles; nor they attacked electrically larger geometries. A different numerical approach is based on the Nystrom-type interpolation techniques, which are efficient for large reflectors up to quasioptical size and multi-reflector configurations [8-10]. In all above mentioned studies the solutions have been performed only for perfectly electric conducting (PEC) zero-thickness surfaces.

As for the imperfect reflectors, so far only resistive ones have been treated with MAR. Here, the E and H-polarization cases have to be regularized in completely different manner. A circularly curved strip with uniform resistivity has been solved in [11] for both polarizations, and a varying-resistivity reflector fed with CSP was solved in [12]. Arbitrary-profile 2-D reflector with resistive surface was treated in [13] for the E-polarization case by using the MAR based on the inverse Fourier transform. Here we will study the H-polarization for the conical-section-profile geometry by using the same technique as developed in [5] for the PEC reflector in the $\mathrm{H}-$ polarization case, i.e. based on the mixed-potential integral equation and RHP inversion.

2. FORMULATION: Cross section of a zero-thickness resistive curved screen symmetrically illuminated by a directive CSP feed is shown in Figure 1. The reflector contour can be elliptic, parabolic or hyperbolic arc with the origin of the used coordinate system taken just in the geometrical focus. Parabolic arc can be considered as a limit case of elliptic arc having the other focus moved to infinity. The feed is considered as a CSP source, which 
produces a directive beam symmetrically looking at the reflector surface. The real-space position of the feed's center of aperture can be arbitrary; in computations, we will take it at the origin, that is at the geometrical focus. The resistivity of reflector surface is assumed uniform and given as $R=Z R_{0}$, where $Z$ is the free space impedance.

When building the solution, the open arc of reflector's contour is completed to the closed contour $C$ by a virtual circular arc having its center on the $x$-axis. Its radius is chosen in such a way that at the junction points the curvatures of the reflector contour and the virtual circle are matched, thus the closed curve has continuous normal needed for the convergence. Another condition for the solvability of the problem is that the branch cut associated with the CSP in the real space must not cross the strip contour $M$. The requirements for the rigorous formulation of the considered boundary-value problem are the satisfaction of the Helmholtz equation, Sommerfeld radiation condition, resistive boundary condition on $M$, and edge condition at its endpoints. The basic mixed-potential integral equation can be obtained by applying resistive surface boundary condition valid in the H-polarization case,

$$
-E_{t}^{i n}(\vec{r})=-Z R_{0} J_{t}\left(\vec{r}^{\prime}\right)+\frac{i Z}{k} \frac{\partial}{\partial l} \int_{M}\left(\frac{\partial}{\partial l^{\prime}} J_{t^{\prime}}\left(\vec{r}^{\prime}\right)\right) G_{0}(\vec{r}, \vec{r}) d l^{\prime}+i k Z \int_{M} J_{t^{\prime}}\left(\vec{r}^{\prime}\right) \cos \left[\xi(\vec{r})-\xi\left(\vec{r}^{\prime}\right)\right] G_{0}\left(\vec{r}, \vec{r}^{\prime}\right) d l^{\prime}, \quad \vec{r} \in M
$$

where $\kappa=\omega \sqrt{\mu_{0} \varepsilon_{0}}$ is the free space wavenumber, $E_{t}^{i n}(\vec{r})=-\left(i Z_{0} / k\right)\left(\partial H_{z}^{i n} / \partial n\right), G_{0}=(i / 4) H_{0}^{(1)}\left(k\left|\vec{r}-\vec{r}^{\prime}\right|\right)$, and $\vec{n}$ is the outer normal unit vector. Suppose now that the arc $M$ can be characterized by parametric equations in terms of the polar angle, $x=x(\varphi), y=y(\varphi)$, where $-\theta_{e} \leq \varphi \leq \theta_{e}$. Besides, we itroduce differential lengths in the tangential direction (the tangential unit vector to the reflector contour is $\vec{t}$ ) at any point on $M$ as $\partial l=a \beta(\varphi) \partial \varphi, \partial l^{\prime}=a \beta\left(\varphi^{\prime}\right) \partial \varphi^{\prime}$, respectively. Here, $\beta(\varphi)=r(\varphi) /[a \cos \gamma(\varphi)], \xi(\varphi)$ is the angle between the normal to $M$ and the $x$-direction, $\gamma(\varphi)$ is the angle between the normal and the radial direction, and $a$ is the radius of the auxiliary circle taken here equal to the focal distance, $f$. Furthermore, $H_{z}^{i n}=H_{0}^{(1)}\left(k\left|\vec{r}-\vec{r}_{c s}\right|\right)$ and $\vec{r}_{c s}=\vec{r}_{0}+i \vec{b}$, where $\vec{r}_{0}$ is the real position vector and $i \vec{b}$ is the complex vector, which characterizes the beam direction and its width (see [3-5] and [9,10] for details).

Note that the integral equation (1) is similar to the PEC arbitrary-profile reflector equation given in [5]. Together with the condition on the current density to be absent off $M$,

$$
J_{t}(\vec{r})=0, \quad \vec{r} \in S,
$$

equation (1) constitutes a dual system. From these two equations, we obtain a dual series equation by using periodicity and projection on the trigonometric polynomials. The details are similar to the PEC case published in [5]. Further this dual series equation is treated with the MAR where analytical inversion is performed using the RHP technique. The final set of algebraic equations is a regularized one, so that its solution exists in the space $l_{2}$ of numerical sequences, and the convergence is guaranteed when solving the truncated equation sets of progressively larger orders.

3. NUMERICAL RESULTS: Numerical results such as the far-field radiation patterns have been obtained for the various reflector contours and various surface resistivities. For small angular size of reflector these results are in agreement with the ones obtained in [12] in the resistive circular-strip case. Besides, the higher directivity and the lower sidelobes are obtained for the parabolic reflector case, i.e. for $e=1$, while much higher sidelobe levels are obtained for the hyperbola and ellipse cases.

4. CONCLUSION: In the development of our earlier studies, arbitrary-profile resistive-surface 2-D reflector antenna has been studied numerically with the MAR technique for the Hpolarization case. Sample results have been obtained and discussed, showing the validity of the technique.

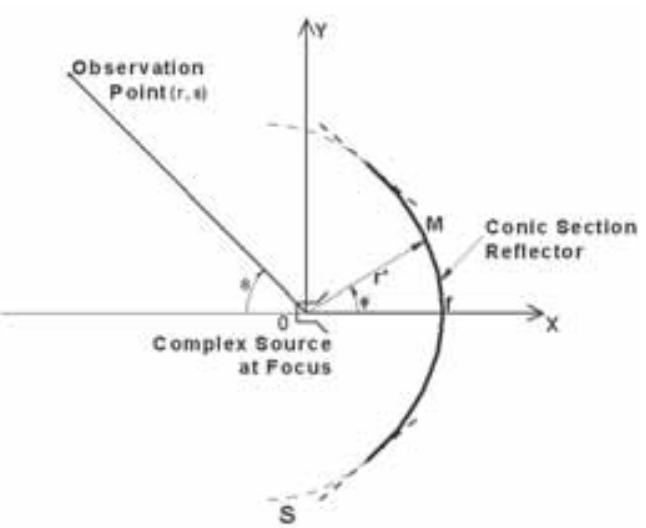

Figure 1: The geometry of the problem 

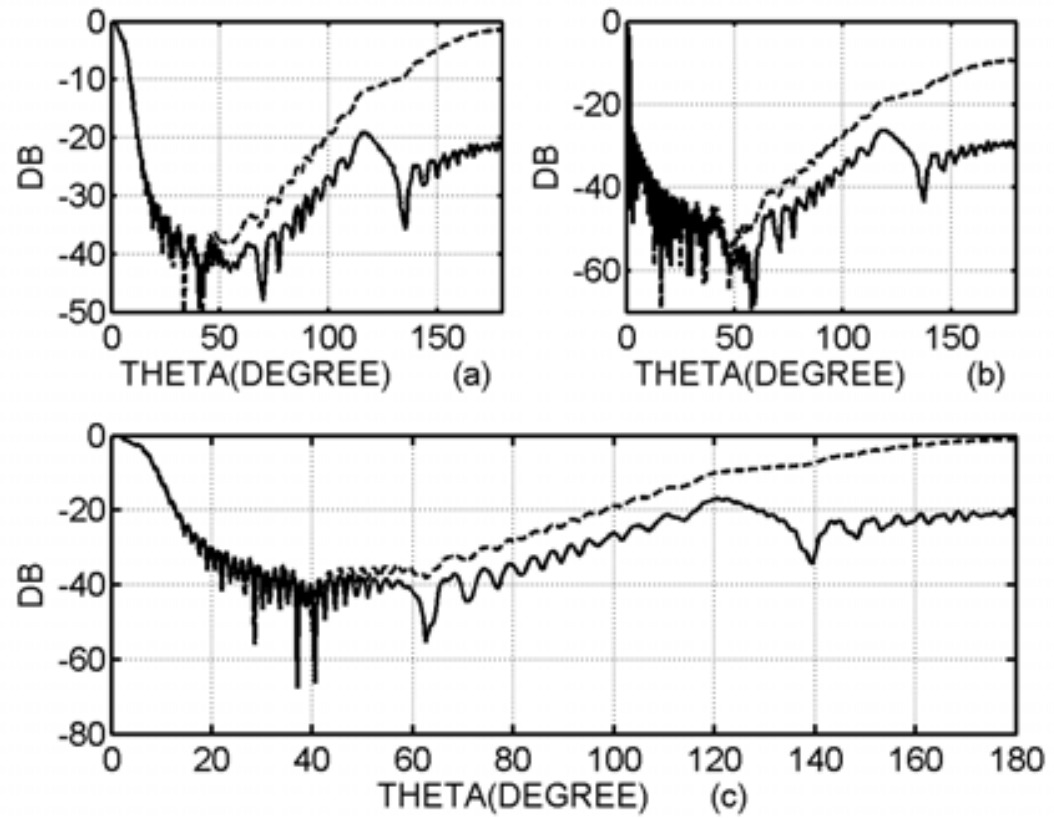

Figure 2: Normalized radiation patterns for different eccentricity factors (a) $e=0.7$, (b) $e=1$, (c) $e=2$. Dashed line is for $R=Z$ and solid line is for $R=0.1 Z$; other parameters are $f=10 \lambda, D=20 \lambda$, and $k b=3$.

\section{References}

[1] M. Idemen, A. Büyükaksoy, "High-frequency surface currents induced on a perfectly conducting cylindrical reflector”, IEEE Trans. Antennas Propagat., vol. 32, no 3, pp. 501-507, 1984.

[2] A.I. Nosich, "Method of analytical regularization in the wave-scattering and eigenvalue problems: foundations and review of solutions" IEEE Antennas Propagat. Magazine, vol. 42, no 3, pp. 34-39, 1999.

[3] T. Oğuzer, A. Altintas, A.I. Nosich, "Accurate simulation of the reflector antenna by complex source-dual series approach” IEEE Trans. Antennas Propagat., vol. 43, no 8, pp. 793-802, 1995.

[4] T. Oğuzer, A.I. Nosich, A. Altıntas "E-polarized beam scattering by an open cylindrical PEC strip having arbitrary conical-section profile”, Microwave Optical Technology Lett., vol.31, no 6, pp. 480-484, 2001.

[5] T. Oğuzer, A.I. Nosich, A. Altıntas, "Analysis of arbitrary conic section profile cylindrical reflector antenna, H-polarization case”, IEEE Trans. Antennas Propagat., vol. 52, no 11, pp. 3156-3162, 2004.

[6] E.I. Veliev, "Numerical-analytical methods of solution of integral equations in two-dimensional diffraction problems", Proc. Int. Conf. Mathematical Methods in EM Theory (MMET*90), Alushta, pp. 73-82, 1990.

[7] Y.A. Tuchkin, "Wave scattering by an open cylindrical screen of arbitrary profile with the Neumann boundary condition", Soviet Physics Doklady, vol. 32, pp. 213-214, 1987.

[8] J. L. Tsalamengas, "Exponentially converging Nystrom's methods for SIEs with applications to open/closed strip or slot-loaded 2-D structures," IEEE Trans. Antennas Propag., vol. 54, no. 5, pp. 1549-1558, 2006.

[9] A.A. Nosich, Y.V. Gandel, "Numerical analysis of quasioptical multireflector antennas in 2-D with the method of discrete singularities", IEEE Trans. Antennas Propagat., vol. 55, no 2, pp. 399-406, 2007.

[10] A.A. Nosich, Y.V. Gandel, T. Magath, A. Altintas, "Numerical analysis and synthesis of 2D quasi-optical reflectors and beam waveguides based on an integral-equation approach with Nystrom's discretization", $J$. Optical Soc. America A, vol. 24, no 9, pp. 2831-2836.

[11] A.I. Nosich, Y. Okuno, T. Shiraishi, "Scattering and absorption of E and H-polarized plane waves by a circularly curved resistive strip", Radio Science, vol. 31, no 6, pp. 1733-1742, 1996.

[12] A.I. Nosich, V.B. Yurchenko, A. Altintas, "Numerically exact analysis of a 2-D variable resistivity reflector fed by a complex-point source”, IEEE Trans. Antennas Propagat., vol. 45, no 11, pp. 1592-1601, 1997.

[13] T. Oğuzer, A. Altıntas, A.I. Nosich, "Analysis of an arbitrary profile reflector antenna system having resistive type surface: E-polarization", Proc. Int. Conf. Mathematical Methods in EM Theory (MMET*06), Kharkov, pp. 181-183, 2006. 